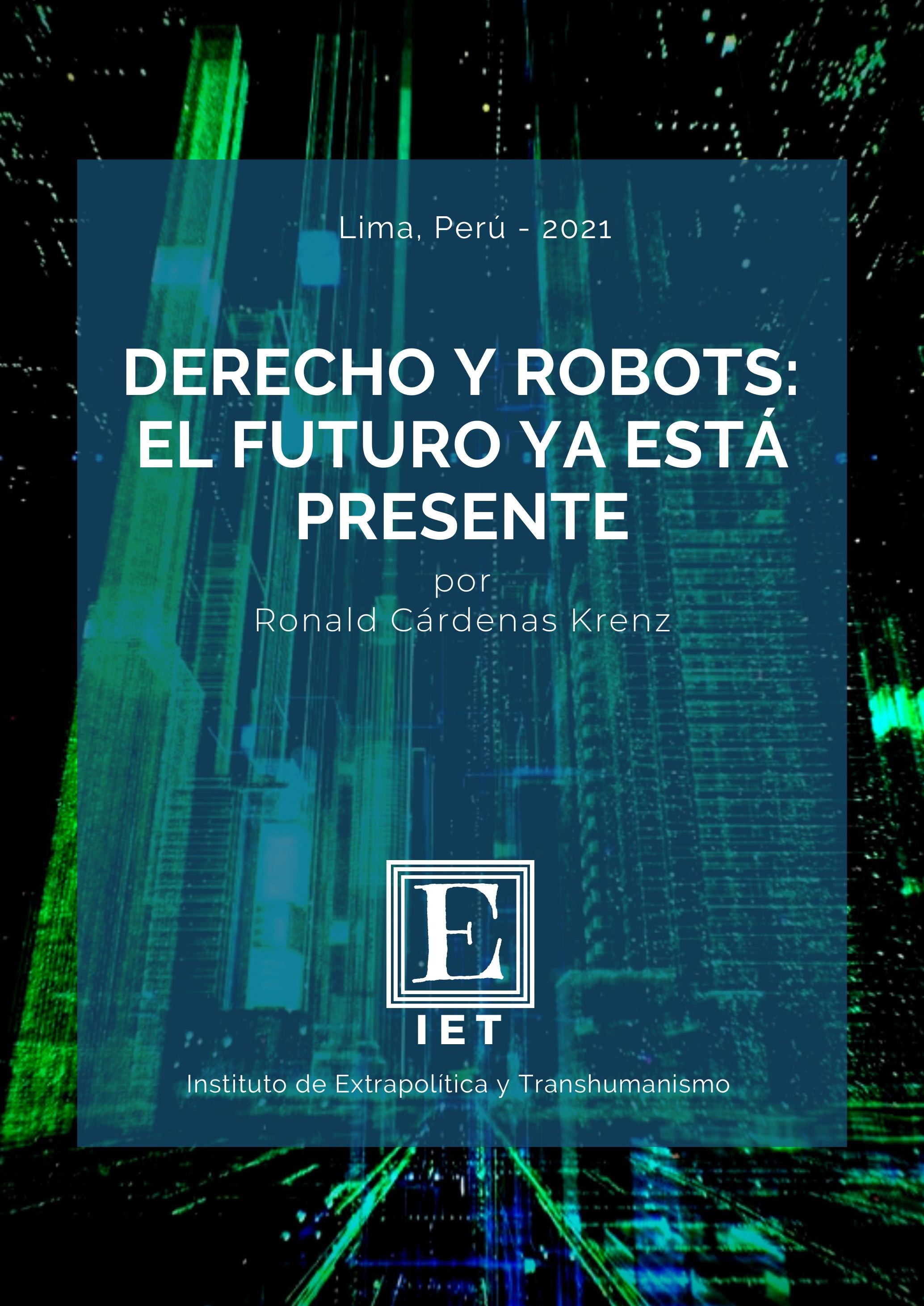




\title{
DERECHO Y ROBOTS: EL FUTURO YA ESTÁ PRESENTE*
}

por

Ronald Cárdenas Krenz

\author{
Sumario: \\ I. Introducción. \\ II. Cuando el futuro se va volviendo cosa del pasado. \\ III. Algunas cuestiones jurídicas vinculadas con el desarrollo de la robótica. \\ IV. Algunas reflexiones vinculadas con el desarrollo de la Robótica. \\ V. Conclusiones. \\ VI. Referencias Bibliográficas.
}

Palabras clave: derecho, ética, robótica, roboética, tecnología

Sobre el autor. Doctorando en Derecho. Magíster en Bioética y Biojurídica. Magíster en Derecho Civil y Comercial. Profesor de la Facultad de Derecho e Investigador del IDIC de la Universidad de Lima. Ex Superintendente Nacional de los Registros Públicos y Presidente del Consejo del Notariado. Miembro de la Academia de Derecho y Ciencias Sociales de Córdoba. Miembro de la Pontificia Academia para la Vida. Miembro del Comité de Ética de la Investigación de la Universidad Femenina del Sagrado Corazón y profesor de la Facultad de Derecho. Profesor en ESAN y de la Maestría en Bioética de la USAT. Ha sido Superintendente Nacional de los Registros Públicos y Miembro del Comité de Ética de la Facultad de Biología de la Universidad Nacional Mayor de San Marcos.

Información de contacto: Rcardena@ulima.edu.pe

\section{iD https://orcid.org/0000-0002-1777-9423}

Cárdenas, R. (2021). Derecho y robots: el futuro ya está presente. Instituto de Extrapolítica y Transhumanismo, 4(1), https://doi.org/10.52749/iet.v4i1.3

* Artículo elaborado para el Instituto de Investigación Científica de la Universidad de Lima. Contó con el apoyo de la Srta. Valeria Padilla Verde, estudiante de la Facultad de Derecho de dicha casa de estudios, quien se desempeñó como Asistente de Investigación. El artículo ha sido republicado con la autorización del autor.

(C) Sociedad Secular Humanista del Perú (SSH).

Fondo Editorial de la Sociedad Secular Humanista del Perú.

Director de Revista: Piero Gayozzo

Publicaciones del Instituto de Estudios Transhumanistas (IET) de la SSH.

Correo: extrapolítica@ssh.orq.pe

Esta obra está bajo licencia internacional Creative Commons 4.0.
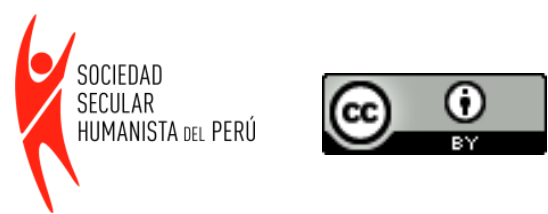


\section{DERECHO Y ROBOTS: EL FUTURO YA ESTÁ PRESENTE}

\section{Introducción}

Las nuevas tecnologías han irrumpido disruptivamente en nuestras vidas: la comunicación se ha vuelto más dinámica e intensa, contratamos de nuevas formas, existen sofisticados tratamientos médicos antes impensados, el entretenimiento se hizo virtual y la educación pasó de la pizarra a la pantalla, entre otros múltiples cambios. Todo ello con el conocimiento como fuente inagotable de recursos. La tecnología no solo ha cambiado el mundo sino también nuestra forma de estar en él.

Pero en la sociedad del conocimiento, como en todo, las virtudes pueden devenir también en riesgos. La información viaja más rápidamente y en mayor escala, pero a la vez somos víctimas de una infodemia en cuya vorágine se mezclan fake news, postverdades, medias verdades, chismes y -a veces- algunas verdades. La indignación ciudadana ha encontrado nuevas y democráticas formas de protesta, disponible las veinticuatro horas del día, ya sea para la denuncia justa como para la acusación ligera, ya sea para el halago o para el agravio.

Con los medios a la mano para exponer hiperbólicamente nuestras vidas, llevados por el egocentrismo, queremos ser protagonistas en la sociedad del espectáculo; y entonces cualquier cosa se puede postear con tal de llamar la atención, de una manera que hasta al propio Oscar Wilde se escandalizaría; llevamos una doble vida, en donde no solo los demás ya no pueden distinguir lo virtual de lo real, sino también nosotros mismos. La vanidad se toma selfies y el valor de la vida parece reducirse al número de likes de la última foto colgada. Narciso ya no se mira al espejo, ahora tiene Instagram o Facebook.

Y así entonces, sabemos más de todos y menos de todo. Tenemos más información, pero menos conocimiento, tejiendo colectivamente una red que ha terminado por atraparnos. Nada importa con tal de estar en línea, sea que ello signifique estar conectados o lucir una buena figura.

En una sociedad orwelliana, Google sería lo más parecido a el Gran Hermano. La intimidad ha cedido el paso a la extimidad.

Seducidos por la tecnología, se habla de edificios inteligentes, autos inteligentes, teléfonos inteligentes, mientras nosotros somos cada vez más ignorantes de una tecnología que nos desborda.

La ingeniería genética ha adquirido dimensiones revolucionarias; ya no son solo pollos con tres piernas, cabras combinadas con ovejas, o perros fosforescentes, pues también existen ya nuevas técnicas de edición como CRISPR-Cas 9, para manipular nuestro genoma como si usáramos un procesador de textos; de esta manera, podremos curar viejas enfermedades, pero también crear otras nuevas; sanar a muchos, pero también comprometer a las próximas generaciones manipulando la propia línea germinal humana.

Como dice Eckman, nos encontramos en un conflicto entre dos ideas: por un lado, la de "We can do it, then we should do it", $y$, por otro, la de "Can does not mandate "ought"”."

En España, se creó hace ya unos años un método para detectar más temprano el síndrome de Down en bebés en gestación a fin que sus progenitores puedan estar mejor preparados para recibirlos; pero, enterados del dato, hoy los padres suelen usarlo para eliminarlos antes de nacer. En una sociedad mercantilista, nadie quiere "productos fallados". 
A tono con las nuevas formas de contratación, compramos vía internet libros, cursos, artículos de primera necesidad o de la última, a un vendedor remoto que puede estar en la esquina, en Estados Unidos o en China. Vamos de compras sin salir de casa, aunque también hay hackers que nos pueden robar desde la comodidad de su sillón.

Drones y satélites nos tienen mejor informados, pero también pueden informar más de nosotros. En nombre de la seguridad, las calles están cada vez más llenas de cámaras; mas a la vez terminamos sacrificando nuestra privacidad o secuestrando la ajena, vulnerando la intimidad del otro con un lapicero, un botón o un reloj cuyas cámaras son suficientes para traerse abajo desde matrimonios hasta presidentes, dándole nuevos alcances al homo videns del que hablaba Sartori.

A propósito de matrimonios, ya hace algún tiempo la tecnología nos planteó la pregunta si podemos ser infieles virtualmente y todavía no nos ponemos de acuerdo con la respuesta. Pero las cosas, en materia de familia, se vuelven más complicadas todavía si vemos cómo se venden bebés creados a la carta, se siguen congelando embriones y cada vez se pone más en entredicho la vieja frase de "madre solo hay una", sobre todo cuando ya es fácticamente posible un bebé creado del óvulo de una mujer, pero con el núcleo de otra, para generar un embrión que cargará en su vientre una tercera, que al final de repente se lo entregue a una cuarta.

En los límites del desborde (si es que no nos hemos pasado ya), está en curso una campaña para promover los "úteros-máquina", promocionándolos como una nueva forma de "liberar" a la mujer. Por otro lado, se ha anunciado ya el envío de una mujer embarazada al espacio para saber "qué pasa" cuando dé a luz allí, sin importar a los organizadores los riesgos para el bebé, pensando solo en futuros viajes espaciales tripulados a lugares más lejanos de nuestra humanidad. Junto con ello, crecen los pedidos para la criogenización, a la que aplican sujetos ansiosos de que se congelen sus cerebros una vez muertos, para que luego sean implantados en la cabeza de otra persona o en una computadora, una vez que contemos con la tecnología para ello. Y, entonces, no sabemos, si la tecnología está para realizar nuestros sueños o para crear impensadas pesadillas.

La definición de la medicina como arte o ciencia para curar, va cediendo peligrosamente su lugar a una medicina del deseo, ofreciendo sus servicios ya no en función de la necesidad sino de la mera frivolidad, incluyendo dentro de sus clientes a preocupadas tías que no tienen mejor idea que regalar a sus quinceañeras ahijadas un par de implantes mamarios por su cumpleaños.

Algunos padres reclaman para que se les permita implantar sensores bajo la piel de sus hijos a fin de monitorearlos. Una modelo anuncia que desea que le implanten un tercer seno. Un científico de Harvard quiere "resucitar" al hombre de Neanderthal. Otra mujer anuncia una vigésima operación para parecerse más a Barbie. Y una pareja sordomuda pide ayuda de la ciencia para tener hijos que nazcan exprofesamente sordos.

La tecnología salta de los laboratorios y consultorios para llegar a las autopistas, de modo que ya vienen circulando a modo de prueba vehículos autónomos, generando nuevos dilemas: ¿cómo programarlos? Si un peatón imprudente se cruza y no hay tiempo para frenar, ¿qué debe hacer el coche? ¿ abrirse a un costado para no atropellarlo, aunque ello signifique chocar contra una pared y acabar con la vida de los pasajeros del auto? ¿O atropellar nomás al peatón para no poner en riesgo a los pasajeros? La gente encuestada sobre el tema, dice que debería programarse para que haga lo primero, pero a la vez esa misma gente preferiría comprar un auto como el segundo.

Antes de que siquiera empecemos a poner en orden algunas ideas, la tecnología está yendo mucho más rápido que la reflexión jurídica. La robótica es uno de esos campos. 
Hoy en día, existen los más sorprendentes tipos de robots, ofreciendo infinitas posibilidades, pero también nuevos riesgos. Y entonces, es importante hacer un alto para detenernos y ver lo que se nos viene, ya sea para un mejor aprovechamiento de las máquinas a efectos del mejor el ejercicio de nuestros derechos, o ya sea para evitar que ellas mismas puedan terminar vulnerándolos. De eso tratan las siguientes páginas.

Soñamos con cyborgs que curiosamente no podrán soñar; y la ciencia ficción es cada vez más ciencia y menos ficción. La tecnología lo va cambiando todo, incluso a nosotros mismos, por lo que habrá qué pensar qué queda de nuestra humanidad y qué hacer para preservarla, antes de que nos convirtamos en un producto más de desecho.

Lo peor -parece ser- no es que los robots se parezcan a nosotros, sino que nosotros nos convirtamos en simples y mecánicos autómatas.

\section{Cuando el futuro se va volviendo cosa del pasado}

Con motivo de la pandemia del coronavirus, los robots fueron utilizados en Wuhan, para repartir alimentos; $y$, en general, en China, la escasez de trabajadores y la necesidad de evitar el contacto personal, llevó a que se incremente el uso de robots en dicho país ${ }^{1}$; además, se han empleado drones y otras aplicaciones algorítmicas para detectar y hacer el seguimiento a personas infectadas con el Covid-19.

En el Perú, Walter Velásquez, un profesor de Huancavelica, creó el robot Qipi, que habla, canta y recita en quechua, ayudándolo a dictar clases en los diversos caseríos hasta donde llegan en plena pandemia del coronavirus.

No cabe duda que la ciencia-ficción es cada vez menos fantasía y más realidad. Los recientes avances en robótica son particularmente notables en la satisfacción de necesidades, deseos o simplemente caprichos, generando enormes expectativas, a la vez que interrogantes.

Mirando al mañana, una de las ideas más recurrentes en el imaginario colectivo, es la de un mundo en donde los seres humanos convivimos con los robots ${ }^{2}$. A partir de allí, se han tejido historias en donde pueden aparecer a veces como héroes, otras como villanos; a veces como fieles servidores del hombre, otras pretendiendo dominarlo, con una serie de matices intermedios entre uno y otro extremo.

En ese elenco futurista, con los robots como protagonistas, desfilan desde HAL de la película "2001: Odisea del Espacio" hasta R2D2 y C3P0 en la saga de "Star Wars", pasando por "Yo Robot" con Will Smith, el robot de la serie "Perdidos en el Espacio", la Robotina de "Los Supersónicos", la niña robot de "La pequeña maravilla", el robot de Mitsuo Suwa en "El hombre par", el robot de "El Hombre Bicentenario" o el memorable C19 del profesor Semo en las fantásticas aventuras de "Fantomas".

Ahora bien, ¿cuál es la realidad en la materia? Un dato interesante es que, en el año 2008, el número de robots industriales en operación era de un millón (Lichocki et al., 2010, p. 39), y si bien estamos todavía lejos de las puestas en escena de Hollywood, podemos decir que el futuro ya empezó. Baste decir que, para el 2019, la Federación Internacional de Robótica (2018)

\footnotetext{
${ }^{1}$ Ver: Diario El Comercio, edición del 21.03.20, p. 10.

2 La palabra "Robot", que viene de la palabra checa robota y significa "trabajo forzado", se origina en una obra teatral checa de ciencia ficción, titulada "RUR Rossum's Universal Robots" (1921), escrita por Karel Capek; aunque su difusión y alcance tiene que ver con la obra "Robot", escrita por Isaac Asimov en 1941 (Martínez, 2018, p. 58).
} 
proyectó la existencia de casi 2'800,000 unidades, previéndose para el 2020 un mercado de robots industriales de cerca de 45 mil millones de dólares.

Ya no solo se trata de máquinas que se encarguen de las tareas más difíciles, meramente mecánicas o riesgosas; ahora podemos encontrarlas como meseras, atendiendo enfermos o recogiendo el polvo de la casa.

Así, en mayo del 2019, en la ciudad de Génova (Italia), el Gran Café Rapallo incorporó a su staff de mozos, nada menos que a dos robots traídos desde China (Che News, 2019), listos para llevar a la mesa los pedidos tomados por los camareros; en Afganistán, la primera camarera robot se llama Timea, habiendo también mozos meseros en diversos locales en Nepal, Japón y China. Ya desde antes, en Estados Unidos, existen restaurantes con robots-barman que preparan excelentes cocteles que incluso saben mejor que los preparados por humanos, teniendo en cuenta la precisión con la que pueden combinar sus ingredientes. Algo más lejos, existe, en un café de Tokio, el robot OriHime-D, que actúa como camarero, siendo la primera cafetería en emplear robots dirigidos a distancia por personas con discapacidades físicas graves.

Se habla también de robots humanoides, los cuales poseen la capacidad de interactuar dinámicamente con nosotros, asemejándosenos en "comportamiento" y aspecto (Núñez, 2019), como es el caso de Zora, que puede ver, oír y desplazarse, visitar a pacientes ancianos y hacer que practiquen ejercicios terapéuticos. Sumado a este, tenemos a Pepper, un robot de compañía que reconoce las emociones fuertes. Además, el robot Nao, permite que un niño enfermo tenga la posibilidad de participar de sus clases desde su cama en el hospital (Pouliquen, 2018, pp. 49 y 50). Existen, además, androides como Geminoid, creado por el japonés Hiroshi Ishiguro.

Una de las más famosas robots hoy en día es Sophia Hanson, de la compañía japonesa Hanson Robotics, una robot humanoide, inspirada en la actriz Audrey Hepburn, diseñada para aprender y adaptarse al comportamiento humano, capaz de dar entrevistas ${ }^{3}$, y que es el primer robot al que se le ha dado ciudadanía, en Arabia Saudita, y sin obligación de andar acompañada de un hombre o de llevar velo, a diferencia de las mujeres de carne y hueso de dicho país.

Dubai tiene ya un policía robot, proyectándose que para el año 2030 , el $25 \%$ de las fuerzas policiales sean compuestas por robots (Shouk, 2017). En Japón, hay un hotel gestionado casi en su totalidad por robots; $y$, en el mismo país, los monjes del templo Kodaiji contrataron hace poco a los mejores especialistas para crear un robot, de 1,95 cm. de alto, que representa a Kannon, "la diosa de la compasión", la cual habla, predica y se desplaza, siendo adorada por sus seguidores, con los que interactúa desde inicios del 2019; también existe el robot Mindar, que recibe a los visitantes de un templo (Amelia Rueda, 2019) y puede interactuar con ellos; aparte, en otro templo, está el pequeño y carismático Xianer (Amalgama, 2016).

Existen ya robots que pueden escribir música, estando en ejecución el proyecto DeepBach, cuyo objeto es crear música al modo de Bach haciendo uso de la inteligencia artificial. De hecho, hoy en día, la inteligencia artificial es ya un recurso para componer canciones.

En materia de pintura, en junio del 2019, en Oxford, Inglaterra, se organizó la primera exhibición de arte de un robot con los dibujos hechos por Ai-Da, una robot con forma humanoide -creada por el artista Aidan Meller- que cuenta con un sistema de inteligencia artificial ${ }^{4}$ que le permite

\footnotetext{
${ }^{3}$ Véase: https://www.youtube.com/watch?v=06hDcL20Vjc ; https://www.youtube.com/watch?v=yEYEJFx-mG4

${ }^{4}$ El término "inteligencia artificial" fue introducido por John McCarthy en 1956, no habiendo un concepto único para definirlo. Para efectos del presente estudio, entendemos por tal al "área de la ciencia informática que se dedica a las máquinas que aprenden por sí mismas" (Frank, et al., 2018, p. 68). Se le puede definir, asimismo, como "una ciencia que trata de estudiar el comportamiento inteligente a través de modelos matemáticos" (Diaz, 2018, p. 14).
} 
reproducir las imágenes capturadas por las cámaras ubicadas en sus ojos, y hablar (CNN, 2019); aparte, existe el algoritmo del proyecto holandés The Next Rembrandt, que es una obra impresa en 3D, que se empleó para recrear cuadros pintados por el famoso artista holandés hace cuatro siglos (Becerra, 2018).

Otro rubro es el de la empresa Boston Dynamics, que ha creado un perro robot llamado Spot, el cual, desde setiembre del 2019, ya se encuentra a la venta, y puede servir para inspeccionar el avance de obras y acciones de patrullaje, entre otros usos; su velocidad es de $10 \mathrm{kph}$, tiene una autonomía de 90 minutos y puede cargar hasta 14 kilos. La misma empresa viene perfeccionando, asimismo, el robot humanoide Atlas, el cual puede hacer parkour y una rutina de gimnasia (Flores, 2019).

Gracias a la inteligencia artificial tenemos también una pequeña pelota: Ballie, que puede servir para cuidar personas, la casa o a nuestra mascota, teniéndonos al tanto de ellos si estamos ausentes; puede incluso hacer llamadas de emergencia, habiendo sido presentada en la feria de consumo electrónico de Las Vegas en enero del 2020.

Por otro lado, existen ya empresas como Real Doll que fabrica maniquíes realistas destinados a satisfacer las apetencias sexuales de los interesados, capaces incluso de responder preguntas que se pueden personalizar a través de una aplicación; los muñecos de plástico con los que cuenta la compañía están debidamente provistos para satisfacer los requerimientos sexuales también de mujeres. De hecho, en Barcelona, existe ya "una" robot llamada Samantha, muñeca sexual de joven apariencia femenina, cuyas medidas son 90-60-90 y está dotada de sensores, prestando sus "servicios" en un prostíbulo.

En el plano deportivo, en la actualidad, el programa Stockfish supera con facilidad a cualquier ajedrecista, existiendo diversos motores de ajedrez que compiten entre sí en campeonatos; lo cierto es que desde que, en 1997, la computadora Deep Blue, de IBM, venciera al campeón mundial de ajedrez Gary Kasparov, las máquinas han incursionado en diversos juegos con éxito: Google cuenta con el programa AlphaGo, que es capaz de entrenarse a sí mismo y que en el 2016 venció a uno de los campeones del mundo en el antiguo juego de mesa chino: Go. La inteligencia artificial también se ha sentado en la mesa de póker y, últimamente, ha incursionado en la pantalla de los videojuegos, siendo capaz de coordinar online con diversos compañeros de equipo hasta diseñar estrategias que le permitan ganar el juego (AnsaLatina, 2019).

No cabe duda de que la tecnología ha avanzado increíblemente desde que Leonardo da Vinci diseñara su "Caballero Mecánico" ${ }^{5}$ y demás está hablar de la presencia de los robots en las fábricas ya desde hace buen tiempo, cumpliendo funciones mecánicas, siendo más bien novedoso su concurso en el área de salud, contándose con un robot como PaPeRo que ayuda en el cuidado de niños.

La aplicación de la robótica en el tratamiento médico de las personas ha generado interesantes resultados. Entre otras aplicaciones, aparte de las ya mencionadas, podemos encontrar las cápsulas médicas inteligentes, el monitoreo de pacientes, el apoyo para los discapacitados, la ayuda en el tratamiento de niños con autismo ${ }^{6}$, etc. Su uso en la medicina es ciertamente

\footnotetext{
${ }^{5}$ Un autómata de tamaño humano, cual caballero con armadura, que podía moverse gracias a un sistema de engranajes y poleas, que puede verse en el museo Da Vinci en Florencia.

${ }^{6}$ En el Perú, un grupo de estudiantes de la ciudad de Huancayo ha creado recientemente Baymax, un robot para tratar a niños

con autismo (y cuyo nombre recuerda a un personaje de Disney de la película "Big Hero 6"); para más detalle, véase: https://caretas.pe/nacional/conoce-a-baymax-el-primer-robot-para-tratar-a-ninos-conautismo-desarrollado-por-huancainos/
} 
prometedor; incluyendo su aplicación en la mejora de la detección del cáncer ${ }^{7}$ y el desarrollo de la medicina personalizada; de esta manera, existe el robot terapéutico PARO; Tug lleva medicamentos a las enfermeras, y MABU le recuerda al paciente cuándo tomar sus pastillas, existiendo otros que acompañan simplemente, como Loomo, constituyendo toda una nueva categoría de robots sociales destinados especialmente para asilos, hospitales o casas para adultos mayores, y cuyas ventas ascendieron a 5.600 millones de dólares en el 2018 (Nugent, 2019). Cabe resaltar que es en el ámbito de robots asistenciales, personales y de servicio en donde hay una mayor interacción entre humanos y robots, lo cual si bien es positivo, nos lleva también a la necesidad de ser conscientes de la posibilidad de riesgo respecto a un posible daño personal (Núñez, p. 63), incluyendo -como lo hemos dicho ya- invasiones a la intimidad.

Por otro lado, científicos de Estados Unidos han utilizado una pequeña sonda robot para recorrer los ventrículos de un cerdo vivo, lo que podría ayudar a detectar problemas cardiacos en seres humanos ${ }^{8}$. Ello aparte de la creación de softwares que permiten una mayor precisión en la interpretación de mamografías o el diagnóstico de diferentes enfermedades. O la aplicación cada vez mayor de Da Vinci, el primer robot cirujano del mundo que se utiliza desde el año 2000 en cirugías de próstata, riñón, estómago y otras. El uso de brazos robóticos para apoyar a los cirujanos, como una extensión virtual de sus manos y ojos, ha permitido acortar el tiempo en las operaciones de próstata, reduciendo el riesgo de sangrado, el peligro de infecciones y disminuyendo el tiempo de internamiento, entre otros efectos positivos (Alcalde, 2016, pp. 199200).

Desde que el médico canadiense Mehran Anvari, introdujo la telemedicina haciendo la primera operación remota de hospital a hospital para realizar una intervención laparoscópica en una paciente a 500 kilómetros de distancia, en donde un sistema de realidad virtual registraba sus movimientos y los trasladaba a un robot sobre el cuerpo de una paciente (Alcalde, 2016, p. 208), sin duda mucha agua ha pasado debajo del puente.

Empero, los usos en el área de la salud no han sido siempre de la forma más apropiada (Cacho, 2018), como sucedió con Ernest Quintana, un paciente de 78 años, a quien a través de un robot con una pantalla de video, se le informó acerca de los resultados de unas pruebas médicas importantes que le habían hecho, en vez de la atención y cuidado especial que el caso ameritaba (Andone \& Moshtaghian, 2019).

En el ámbito empresarial, hoy por hoy, ya existen robots que toman decisiones en las empresas, como lo ha resaltado la revista Forbes (Vallarino, 2015). En materia de finanzas, las compañías de gestión de activos están empezando a introducir robo-advisors, ofreciendo servicios de asesoría financiera, los cuales requieren poca o ninguna intervención humana, cometiendo menos errores y cobrando comisiones más bajas (Rouhiainen, 2018, p. 59). Cabe decir que, en el año 2015, seis de los ocho principales fondos de alto riesgo de Estados Unidos, gracias a algoritmos derivados de la inteligencia artificial, ganaron alrededor de 8000 millones de dólares. También existen empresas como Betterment, de Jon Stein, quien a sus mediados treinta años, convirtió a su empresa en líder mundial en el campo de robots asesores para la asesoría financiera, los cuales atienden durante las 24 horas del día y a un precio más módico que los asesores tradicionales (Frank et al., 2018, pp. 16, 20).

Se habla ahora incluso de una "Tecnología Social", en la cual las máquinas ya no solo nos ayudan a hacer más cómodas nuestras vidas, automatizar algunas cosas o a bajar los costos de producción, sino que también pueden cumplir un papel importante en la defensa de los derechos humanos. De esta manera, por ejemplo, hoy se usan drones para repartir vacunas y

\footnotetext{
${ }^{7}$ Existen ya, por ejemplo, sistemas de inteligencia artificial capaces de superar a los humanos en la predicción del cáncer de mama (Mayer et al., 2020).

${ }^{8}$ Diario El Comercio. Lima: edición del 26.04.19, p. 19.
} 
medicinas en lugares de difícil acceso. Vanuatu es un país ubicado en el Pacífico Sur, conformado por pequeñas islas a las que es complicado llegar, por lo que, en diciembre de 2018, UNICEF firmó un contrato con el Ministerio de Salud de dicho país para usar drones a fin de entregar vacunas. A su vez, en Ghana, en mayo del 2019, se aprobó la incorporación de drones al sistema público sanitario para el envío de medicinas y sangre a todo el país (The Technolawgist, 2019).

La variedad de aplicaciones es amplísima: Un robot bombero tuvo protagonismo durante el incendio de la catedral de Notre Dame en el 2019; existen tenedores "inteligentes" ${ }^{9}$, un robot que orienta a los pasajeros en el aeropuerto de Munich ${ }^{10} y$ hasta un robot diseñado por Samsung para llevar papel higiénico.

En el Perú, la robótica viene ingresando a las organizaciones tanto como opción de hardware como de software, ya sea a través del uso de brazos mecánicos o el procesamiento de datos, siendo de mencionar que el $20 \%$ de las empresas de nuestro país usan robots/chatbots, $14 \%$ ya utiliza inteligencia artificial y un $10 \%$ emplea drones, previéndose un alza exponencial en la robótica en los años siguientes ${ }^{11}$. Existe también un robot diseñado para recoger desperdicios en la playa: Sullkapata, diseñado en el Perú por el ingeniero Miguel Siguenza, el cual puede recoger objetos de menos de cuatro centímetros, como también el ya mencionado Bayman ${ }^{12}$, creado por un grupo de estudiantes para ayudar a niños con trastorno del lenguaje.

Dentro de los logros más sorprendentes, han empezado a aparecer robots que pueden tener programado un "libre albedrío" artificial, de modo que, entre varias opciones, sus circuitos "eligen" la opción más adecuada (Golombek, 2013, p. 181). Existen ya, en Estados Unidos, robots que tienen cierta autoconsciencia ${ }^{13}$. Se trata de sistemas robóticos que tienen una capacidad de autoaprendizaje, pero que incluyen un componente negativo: la generación de reacciones imprevisibles, con comportamientos o interacciones inesperadas, y con una pérdida de control o dirección por parte de su creador, propietario o programador (Núñez, pp. 12 y 13).

Los avances en el estudio del cerebro y la aplicación de la tecnología, vienen promoviendo la creación de interfaces cerebro-ordenador ( $\mathrm{BCl}$ según sus siglas en inglés), que tratan de abrir un canal de comunicación que contacte al cerebro con un dispositivo externo, ya sea una prótesis, un brazo robótico o una computadora (Viosca, 2018, p. 39), pudiendo permitir entonces a una persona que ha tenido un accidente, volver a tener la capacidad de sentir, moverse o hablar. En diciembre de 2012, la revista británica The Lancet informó que investigadores estadounidenses han creado un brazo artificial que puede ser movido con el pensamiento. Por otro lado, la Universidad de Montfort, en Inglaterra, ha desarrollado un videojuego que se controla con la vista, pensando en los niños con discapacidad severa que no pueden usar un teclado.

Pero los alcances de estas interfaces pueden ser más sorprendentes, pues ya se habla de la posibilidad de contar con máquinas que pueden deducir lo que una persona quiere, permitiendo una comunicación neural directa (Andersen, 2019, p. 24).

Vivimos en un mundo en donde nos servimos y dependemos cada vez más de la inteligencia artificial, a través de artefactos diseñados para superar nuestras propias limitaciones físicas y

\footnotetext{
${ }^{9}$ Hapifork ha creado el tenedor inteligente que avisa al comensal si está comiendo demasiado rápido y mide la frecuencia con que nos llevamos la comida a la boca, entre otras aplicaciones.

${ }^{10}$ En febrero de 2018, en el aeropuerto de Munich, empezó a brindar servicios de orientación el robot llamado Josie Pepper, en la Terminal 2 de dicho aeropuerto, siendo la primera prueba de un robot humanoide equipado con inteligencia artificial que atiende en un aeropuerto alemán. Más detalles sobre el particular, puede encontrarse en la propia página web del aeropuerto: https://www.munichairport.com/hi-i-m-josie-pepper-3613413.

${ }^{11}$ Fuente: Suplemento Día 1. El Comercio, edición del 06.05.19, p. 16.

12 Suplemento El Dominical del diario El Comercio. Lima: edición del 31.03.19.

${ }^{13}$ Diario El Comercio. Lima: edición del 06.02.19, p. 15.
} 
mentales ${ }^{14}$. Estos avances nos permiten manejar un número mayor de datos, a mayor velocidad, y sobre la base de sofisticados modelos de redes, aparte de permitirnos hacer cosas que por nuestros propios medios no podríamos, en un desarrollo tecnológico espectacular.

La tecnología digital es parte fundamental de nuestra vida ordinaria y contamos entonces con aparatos que nos indican cómo llegar a un lugar (GPS), que nos guían entre el tráfico y hasta nos avisan si hay un policía en el camino (Waze), que nos recomiendan qué películas y series ver (Netflix), que seleccionan las noticias de nuestros amigos que más nos interesan (Facebook), que pueden atender diversas cosas de la casa (Alexa), hacer llamadas por nosotros (Siri) o hasta ayudarnos a encontrar pareja (Tinder).

Hemos abierto las puertas a diversas formas de inteligencia artificial porque hacen nuestra vida más cómoda y agradable, integrándose a nuestro día a día hasta llegar a pasar casi desapercibidas (Frank et al., 2018).

Inventamos las calculadoras para hacer cálculos complejos que nos era complicado realizar, y ahora les dejamos a ellas hasta los cálculos más sencillos, tanto que hasta ya hemos olvidado cómo se hacían. Si antes ejercitábamos nuestra memoria guardando los números telefónicos de nuestros seres queridos, ahora dejamos la tarea al celular y sin él no somos capaces de llamar a casi nadie. Podemos conversar a través de las redes sociales con una persona en cualquier lugar del mundo, pero paradójicamente hablamos menos con quien tenemos enfrente. $Y$ hasta hay quienes piensan en para qué estudiar si Google de inmediato tiene todas las respuestas.

Sin querer, las máquinas controlan o determinan aspectos sustanciales de nuestras vidas más allá de lo que estamos dispuestos a reconocer ${ }^{15}$. Y ya no solo nosotros nos conectamos con ellas, ahora también ellas se conectan entre sí, a través del internet de las cosas, prescindiendo de nosotros inclusive: así, por ejemplo, en medio del tráfico, contamos con una aplicación que recoge la información de diversos teléfonos que se conectan con un sistema de satélites a través de un ordenador central que procesa la información para decirnos la mejor ruta para llegar a nuestro destino, de esta manera: "Estamos controlados, supervisados, aconsejados, informados, condicionados por ordenadores que hablan con otros ordenadores" (Latorre, 2019, p. 72).

Vale decir que se estima que, para el 2020, los dispositivos interconectados en todo el mundo podrían oscilar entre 50000 millones y 200000 millones (Frank et al., 2018).

Pero las oportunidades no vienen solas, pues suelen llegar de la mano con riesgos, como el caso del accidente del robot que fue atropellado por un vehículo autónomo en una feria en Las Vegas en el 2019 o, el atropello de una persona por un vehículo autónomo. Pero existen otros usos más complejos y delicados, como es el empleo de drones y Robot Killers para matar a un enemigo a distancia cuyos usos pueden atentar contra el Derecho Internacional Humanitario.

Sea como sea, lo cierto es que los robots han llegado del futuro para hacerse cada vez más presente en los más diferentes ámbitos de nuestras vidas, planteando enormes posibilidades, pero también complejos y formidables retos para el pensamiento jurídico.

\footnotetext{
${ }^{14}$ Mientras que, sacar la cuenta del restaurante nos toma unos 3 minutos, existen ya superordenadores que realizan más de un millón de billones de operaciones por segundo, lo que equivale a un uno seguido de quince ceros (Latorre, 2019, p. 50).

${ }^{15}$ Señala Marcos Saudoy: "We think we're in control, and at the moment, we're not. And unless we learn the ways that we're being pushed and pulled around by algorithms, we're going $t$ be at their mercy" (Harris, 2019).
} 


\section{Algunas cuestiones jurídicas vinculadas con el desarrollo de la robótica}

Ya pueden hacerse notar algunos temas de discusión, los cuales no vamos a desarrollar aquí ya que excederían el objetivo y extensión del presente artículo, pero que es oportuno enlistar, ya que el derecho tendrá que analizarlos y pronunciarse sobre ellos.

La pregunta acaso más importante es si los avances en robótica deben llevarnos a considerar a los robots como sujetos de derecho, como ya lo vienen planteando algunos, hablándose de otorgarles un estatuto jurídico especial, promoviendo su consideración como personas electrónicas; aunque también ha sido calificado por otros como una aberración; cabe mencionar que existe una Resolución del Parlamento Europeo, del 16 de febrero del 2017 en la que reconoce que cabría hablar (aunque sea) a largo plazo de una personalidad jurídica específica para los robots.

Al margen de que no sean sujetos de derecho, queda la cuestión de qué hacer con un robot que sea utilizado para cometer o ayudar a cometer un crimen. ¿Podría haber sanciones que recaigan sobre el robot y obligarlo, por ejemplo, a realizar trabajos forzados o a desconectarse?

Asumiendo que son objetos, la pregunta es quién responde por lo que hagan: ¿el programador, el fabricante, el vendedor, el comprador o el Estado que acepta su comercialización? El sistema clásico de la responsabilidad civil tendrá que repensarse, pues la realidad lo pondrá en aprietos, debiendo de descartarse previamente si tras las propuestas del reconocimiento de la responsabilidad de los robots exista en algún caso una manipulación tendenciosa para liberar de responsabilidad a los fabricantes o a otras personas (Rogel, 2018, pp. 21-22).

Aunado a ello está el tema del uso de robots para actividades de control y seguridad ciudadana, ¿podríamos usarlos para reprimir una manifestación aunque ello signifique el uso de la fuerza? ¿podríamos programarlos para identificar a un delincuente que está libre y detenerlo en cuanto lo encuentre?

A fin de reducir el número de entuertos que retarán a los jueces, será importante definir los criterios que deben regir la programación de robots; en este punto, más allá de las reglamentaciones legales, o precisamente por la imposibilidad de ellas para cubrirlo todo, será fundamental el desarrollo de la que ha venido a llamarse una "roboética", es decir, una ética vinculada con los robots.

Demás está decir la necesidad de repensar el tema laboral en la medida que los robots van incursionando cada vez más en el área del empleo. De hecho, su presencia ha de significar la reducción de diversos puestos de trabajo en las empresas (casos conocidos son, por ejemplo, los de Apple, Samsung y Dongguan). Vale decir que un estudio de Oxford Economics de agosto del 2019, calcula que los robots han acabado con 400.00 empleos netos en Europa desde el 2000 (ABC, 2019). Sin embargo, a la vez, esta incorporación de los robots al ámbito laboral implicará la aparición de nuevos empleos para las personas, menos manuales y más intelectuales, menos mecánicos y más productivos. Vinculado con este tema, como una propuesta para paliar el desempleo que ocasionarán los robots, se ha planteado la posibilidad de establecer un impuesto sobre éstos, metiendo de esta manera en el lío de su regulación también a los tributaristas.

Visto que los robots pueden ahora componer, pintar o hacer retratos, una discusión particularmente compleja es la que tiene que ver con los derechos de autor. Si bien suena poco racional imputar a un robot la autoría de una obra, parece también arbitrario imputársela al dueño del robot pues no fue él quien en realidad pintó el cuadro. Menudo problema.

Adicionalmente, no quedará duda que deberá revisarse los derechos vinculados con la guerra para intentar regular de alguna manera el uso de robots en el transcurso de conflictos bélicos, 
como también deberán revisarse los criterios para definir la infidelidad en la pareja cuando la esposa se entere que su marido la engaña con una robot.

\subsection{Posibilidades específicas que ofrece la inteligencia artificial en el ejercicio y aplicación del Derecho}

En el ámbito legal, la inteligencia artificial ofrece innumerables posibilidades como la posibilidad de contar con una plataforma que permita revisar la jurisprudencia, leyes y doctrina en forma rápida y más integral, así como el historial de los propios magistrados y abogados que son parte de un proceso.

Ya se habla en el Derecho, de "roboabogados" ${ }^{16}$, estando en plena aplicación sistemas informáticos que la emplean para la mejor ejecución de procesos de Due Diligence (Frank et al., 2018). A su vez, JP Morgan Chase \& Co. cuenta con un programa de aprendizaje automático que ha suprimido más de 360000 horas de trabajo al año que antes realizaban abogados (Rouhiainen, 2018, p. 60).

En la Universidad de Cambridge, un grupo de estudiantes de Derecho ha desarrollado la startup Case Crunch, la cual, integrando el derecho con la i.a., permite anticipar el resultado de un proceso en forma más eficiente que los estudios de abogados, lo que se ha corroborado en una competencia ad hoc en donde mientras los letrados tuvieron una eficacia del $66.3 \%$ en la predicción de los casos, los robots alcanzaron un $86.6 \%$ de aciertos ${ }^{17}$.

En un paso más audaz, en Estonia, desde el año 2019, el Ministerio de Justicia de dicho país -a la vanguardia en el tema- viene trabajando en una inteligencia artificial que actúe como juez, para encargarse de los casos sencillos, sobre reclamos menores, aun cuando la decisión final igual estaría siempre a cargo de un juez. El robot podría analizar miles de documentos legales y cruzar información para resolver, con el consiguiente ahorro de tiempo (Connelly, 2019) (The Technolawgist, 2019).

En Colombia, existe Siarelis, que es un sistema que puede ayudar al juez en sus proyectos de resolución y absolver consultas de las partes, ahorrándole horas de trabajo y dándole agilidad a los procesos (Guauque, 2018).

En Estados Unidos, existe ROSS, que responde preguntas jurídicas, da cuenta de juicios pasados y de las últimas novedades jurídicas en internet (Scaliter, 2016). A su vez, Public Safety Assestment es un sistema que ayuda a establecer fianzas o calcular el riesgo de decisiones judiciales (Latorre, 2019, p. 143).

Aparte de las ventajas de una mayor celeridad y manejo de información, la tecnología ofrece, junto a la posibilidad de contar con un servicio de justicia más predecible, el beneficio de eliminar la corrupción y las influencias sospechosas, contando con un sistema jurídico más neutro e independiente.

Finalmente, ante posibles errores o "falta de criterio" de los sistemas informáticos, o cuestiones que exijan una sensible capacidad de ponderación, podría incluirse siempre la posibilidad de apelar a una última instancia humana, como ya se viene planteando en la doctrina (Latorre, 2019, p. 141).

\footnotetext{
${ }^{16}$ Se trata de programas informáticos capaces de buscar en internet información diversa de interés, brindando ayuda legal (Kaku, 2018, p. 148).

17 Para mayor detalle al respecto, véase: http://www.elfinanciero.com.mx/tech/los-robots-quierenganarle-a-los-abogados-y-\%20lo-estan-logrando?fbclid=IwAR3owcW3hEKfaNmus4KjROvNIZPQRNMBOfI
} 


\section{Algunas reflexiones vinculadas con el desarrollo de la robótica}

El debate científico está abierto. Así como hay quienes piensan que será posible contar en el futuro con robots que tengan conciencia como nosotros, hay quienes piensan que ello es imposible.

En todo caso, en este indetenible proceso, como dice Latorre, si creamos máquinas que nos superen intelectualmente, habrá que pensar en cuál es el lugar que nos queda a nosotros (Latorre, 2019, pp. 11-12).

Algo que resulta claro, es que, pudiendo ser tan hábiles a la hora de sacar cálculos, carecen sin embargo de algo tan obvio para nosotros como es el sentido común. Y entonces, son torpes para desplazarse por una casa, entender que el tiempo no va para atrás, o que las cuerdas son para tirar mas no para empujar (Kaku, 2010, pp. 141, 145).

En todo caso, quizás sea necesario reparar en que los robots no podrán ser como nosotros porque ni el ordenador es un cerebro, ni el cerebro es un ordenador.

No somos meros impulsos eléctricos sino también experiencias; no solo somos razón sino también sentimientos, no solo estamos condicionados por nuestra física y nuestra química, sino que también somos fruto de nuestra libertad. Somos la síntesis de una singular experiencia subjetiva, y ella demanda, "una capacidad de examinar y un razonamiento abstracto propio del espíritu" (Jouve, 2015).

\subsection{Una tarea necesaria}

Existen ya algunos instrumentos internacionales que se van aprobando. Europa cuenta con una Resolución del Parlamento Europeo, del 16.02.17, con recomendaciones para la Comisión sobre normas de Derecho Civil sobre Robótica. También es interesante mencionar que la Comisión Europea ha presentado el 19.02.30 una Comunicación al Parlamento Europeo, al Consejo, al Comité Económico y Social Europeo y al Comité de las Regiones, titulada «Dando forma al futuro digital de Europa».

Sería conveniente entonces empezar a trabajar en la región en un marco legal que nos permita contar con el andamiaje jurídico necesario para la más debida recepción de las nuevas tecnologías en robótica, anticipándonos a futuros escenarios.

\section{Conclusiones}

1.- La robótica y la inteligencia artificial han tenido avances extraordinarios en los últimos tiempos, poniéndonos en los inicios de una revolución. Han llegado para quedarse y nuestro derecho debe prepararse para recibirlas.

2.- Si bien en una gran cantidad de aspectos específicos los robots superan ya ampliamente a los seres humanos, desde una perspectiva integral no puede compararse la inteligencia artificial con la inteligencia humana. Los valores no se pueden programar. Los robots no tienen conciencia. Un ordenador nunca podrá ser un cerebro, pues el cerebro no es un ordenador.

3.- Desde una perspectiva social y jurídica, dadas las fantásticas posibilidades que puede ofrecer la robótica para el beneficio de la sociedad, ella debe desarrollarse de la mano de una 
robo-ética y con un marco jurídico básico, hoy inexistente.

4.- El desarrollo de la robótica plantea diversas cuestiones jurídicas en diferentes ámbitos del derecho, lo cual requiere de desarrollos específicos en cada disciplina jurídica, debiendo tenerse siempre en consideración el respeto de los Derechos Fundamentales de la persona. La regulación de los vehículos autónomos se presenta como el terreno en donde empezarán estos debates y se irán adoptando las primeras pautas fundamentales sobre la materia.

6.- No podemos exigir conductas éticas a los robots, pero sí a quienes los programan y a quienes los comercializan.

7.- Así como el Derecho puede orientar el desarrollo de la robótica en el cauce del respeto de los derechos, la inteligencia artificial puede ser aprovechada por el Derecho para facilitar la tarea de jueces y abogados.

\section{Referencias}

ABC. (26 de Agosto de 2019). Los robots arrasan 400.000 empleos netos en Europa desde el año 2000. Obtenido de https://www.abc.es/economia/abci-robots-arrasan-400000empleos-netos-europa-desde-2000-201908261316 noticia.html

Alcalde, Jorge (2016). ¿Por qué los astronautas no lloran? La gran ciencia de las pequeñas cosas. Barcelona: Planeta.

Amalgama. (20 de Mayo de 2016). Xianer un monje robot que une tecnología y budismo [Video de YouTube]. Obtenido de https://www.youtube.com/watch?v=nWEKcDNQESI

Amelia Rueda. (14 de Agosto de 2019). Mindar: el robot predicador [Video de Youtube]. Obtenido https://www.youtube.com/watch?v=Z7kLgwAVaeQ\&ab channel=AmeliaRueda

Andone, D. y Moshtaghian, A. (10 de marzo de 2019). Un médico usó un robot con pantalla para decirle a un paciente que iba a morir, la familia del hombre está molesta. CNN. Recuperado de: https://cnnespanol.cnn.com/

AnsaLatina. (01 de Junio de 2019). Otra derrota humana ante inteligencia artificial. Obtenido de http://www.ansalatina.com/americalatina/noticia/tecnologia/2019/06/01/otraderrota-humana-ante-inteligencia- artificial 7f5c0283-961b-4f1c-aba4086076a25a3e.html

Becerra, J. (14 de junio de 2018). Por qué las máquinas nunca podrán crear arte. El Mundo. Recuperado de: https://www.elmundo.es/

Che News. (05 de Mayo de 2019). Rapallo, al Gran Caffè arrivano due nuovi camerieri: sono robot. Obtenido de https://www.chenews.it/2019/05/05/rapallo-gran-caffe-camerierirobot/

CNN. (07 de Junio de 2019). Conoce a Ai-Da, el primer robot que presentará una exposición de arte. Obtenido de https://cnnespanol.cnn.com/video/robot-ai-da-inteligenciaartificialdibujar-oxford-pkg-digital-original/

Díaz Alabart, S. (2018). Robots y responsabilidad civil. Madrid: Editorial Reus. Pág. 7.

Flores, C. (25 de setiembre del 2019). Spot, el perro robot de Boston Dynamics, comienza a fabricarse en masa y ya es posible comprarlo. El Economista. Recuperado de: https://www.eleconomista.es

Frank, M., Roehrig, P. y Pring, B. (2018). Qué haremos cuando las máquinas lo hagan todo. Madrid: LID Editorial Empresarial.

Golombek, D. (2013). Cavernas y Palacios. Pág. 181. Siglo Veintiuno Editores 
Guauque, C. (10 de Noviembre de 2018). El robot que entró al equipo de la Supersociedades para asistir en pleitos mercantiles. Obtenido de https://www.semana.com/nacion/articulo/supersociedades-integro-a-un-robot-paraasistir-a-los-jueces-en-pleitos-mercantiles/590406/

Harris, J. (05 de marzo de 2019). Could robots make us better humans? The Guardian. Recuperado de: https://www.theguardian.com/international

Latorre, J. I. (2019). Ética para máquinas. Barcelona: Editorial Planeta

Lichocki, Pawel. Kahn, Peter \& Billard, Aude. (2011). The Ethical Landscape of Robotics. IEEE Robotics \& Automation Magazine, (Abril de 2011). At. 39.

Martínez Mercadal, Juan José. Vehículos autónomos y derecho de daños. Revista de la Facultad de Ciencias Económicas de la Universidad Nacional del Nordeste. Octubre de 2018. At. 55.

Kaku, M. (2010). Física de lo Imposible. Debolsillo.

Kaku, M. (2018). El futuro de la humanidad. Penguin Randomm. House Grupo Editorial.

Núñez, M. (2019). Inteligencia artificial y responsabilidad civil. Madrid: Editorial Reus.

Pouliquen, T. (2018). Transhumanismo y fascinación por las nuevas tecnologías.

Madrid: Ediciones RIALP, S.A.

Rogel Vide, Carlos et al. (2018). Los robots y el Derecho. Madrid: Editorial Reus.

Shouk, Al. (13 de marzo de 2017). First robot cop to join Dubai Police by May, official says. Gulf News. Recuperado de: https://gulfnews.com/

The Technolawgist. (12 de Junio de 2019). Estonia se prepara para tener "jueces robot" basados en inteligencia artificial. Obtenido de https://www.thetechnolawgist.com/2019/06/12/estonia-se-prepara-para-tenerjueces-robot-basados-en-inteligencia-artificial/

The Technolawgist. (06 de Mayo de 2019). Los drones que salvan vidas repartiendo vacunas y sangre. Obtenido de https://www.thetechnolawgist.com/2019/05/06/los-drones-quesalvan-vidas-repartiendo-vacunas-y-sangre/

Vallarino, D. (17 de Agosto de 2015). Llegaron los robots que toman decisiones en las empresas. Obtenido de Forbes: https://www.forbes.com.mx/llegaron-los-robots-que-tomandecisiones-en-las-empresas/

Viosca, J. (2018). El cerebro. Descifrar y potenciar nuestro órgano más complejo. Pág. 121. National Geographic

Scott Mayer Mckinney ET AL. International evaluation of an Al system for breast cancer screening. Nature. January 1, 2020. At. 89. 